\begin{tabular}{|c|c|}
\hline Title & High-Speed In Situ Observation System for Sonoporation of Cells With Size- and Position-Controlled Microbubbles \\
\hline Author(s) & Kudo, Nobuki \\
\hline Citation & $\begin{array}{l}\text { IEEE transactions on ultrasonics ferroelectrics and frequency control, 64(1), 273-280 } \\
\text { https://doi.org/10.1109// UFFC.2016.2606551 }\end{array}$ \\
\hline Issue Date & 2017-01 \\
\hline Doc URL & http:/hdl.handle.net/2115/66187 \\
\hline Rights & $\begin{array}{l}\text { (c) } 2016 \text { IEEE. Personal use of this material is permitted. Permission from IEEE must be obtained for all other uses, in } \\
\text { any current or future media, including reprinting/republishing this material for advertising or promotional purposes, } \\
\text { creating new collective works, for resale or redistribution to servers or lists, or reuse of any copyrighted component of } \\
\text { this work in other works. }\end{array}$ \\
\hline Type & article (author version) \\
\hline File Information & kudo- uffc.pdf \\
\hline
\end{tabular}

Instructions for use 


\title{
High-speed in Situ Observation System for Sonoporation of Cells with Size- and Position-Controlled Microbubbles
}

\author{
Nobuki Kudo, Member, IEEE
}

\begin{abstract}
A high-speed in situ microscopic observation system developed for basic studies on mechanisms of sonoporation is introduced in this paper. The main part of the system is an inverted-type fluorescence microscope, and a high-speed camera of $20 \mathrm{MHz}$ in maximum framing rate was used to visualize dynamics of cavitation bubbles that causes a sonoporation effect. Differential interference contrast and fluorescence techniques were used for sensitive visualization of cell changes during sonoporation. The system is also equipped with optical tweezers that can move a microbubble of several microns in size by using a donut-shaped light beam. In situ microscopic observation of sonoporation was carried out using a cell with a size- and position-controlled microbubble. The experimental results showed that the ability of cells to repair sonoporation-induced damage depends on their membrane tension, indicating the usefulness of the observation system as a basic tool for investigation of sonoporation phenomena.
\end{abstract}

Index Terms-Biological effects \& dosimetry, Biophysical Mechanisms, Therapeutics, Ultrasound contrast agents.

\section{INTRODUCTION}

$\mathrm{S}$ ONOPORATION is a technique to induce a temporal increase in cell membrane permeability by ultrasound exposure and transport drugs or genes that normally have no permeability into cells [1]-[3]. Early studies on sonoporation showed that ultrasound exposure in the presence of microbubbles greatly improves sonoporation effects [4], [5], suggesting that mechanical effects of oscillating cavitation bubbles on cells are responsible for sonoporation.

Continuous or long-tone-burst ultrasound is generally used to obtain higher efficacy of sonoporation. Since cavitation bubbles have highly random and complex activities under this exposure condition, it is impossible to predict the place and timing of a cavitation bubble to damage the cell membrane, and thus in situ microscopic observation of sonoporation

Manuscript received April XX, 2016; revised YYY ZZ, 2016. This work was supported in part by the Japan Society for the Promotion of Science 23300182, 25560233 and R \& D group of Japan Society of Ultrasonics in Medicine.

N. Kudo is with the Graduate School of Information Science and Technology, Hokkaido University, Sapporo 060-0814, Japan (e-mail: kudo@bme.ist.hokudai.ac.jp). phenomena is difficult. In a previous study, we showed that exposure of cells to single-shot short pulsed ultrasound can induce a sonoporation effect if preexisting bubbles are attached to the cells [6]-[8]. In this situation, in situ microscopic observation of sonoporation phenomena is possible because the short duration of the pulse can limit the range of bubble movement inside a field of view and enables prediction of the sonoporation site.

Acoustically induced cavitation causes various types of biological effects [9][10], and observation of cavitation dynamics during sonoporation phenomena is a direct method for elucidating bubble activities and interaction of bubbles with cells. Ultrasound of several $\mathrm{MHz}$ in center frequency and microbubbles of several microns in diameter are typically used for sonoporation; therefore, microscopic observation at framing rates higher than the ultrasound frequency is desirable to visualize bubble dynamics [7], [8], [11]-[13] and bubble-cell interaction [6], [14]-[18]. Bright-field (BF) microscopy is suitable for observation of bubble activities, and florescence microscopy combined with differential interference contrast (DIC) techniques is essential for sensitive detection of cell changes.

Understanding of sonoporation phenomena is, however, still difficult because cultured cells have large variance in their shape and maturity, and bubbles also have large variance in their size and relative position to the cells. These variances cause a large difference in the sonoporation effect of individual cells and make it difficult to study the mechanisms and find optimum conditions for sonoporation.

To overcome the limitations in conventional sonoporation studies, we have developed a system that is equipped with optical tweezers that can move bubbles of several microns in size [19]. Using the optical tweezers, a bubble of a desired size is selectively picked up from bubbles of various diameters and moved to a desired position adjacent to a cell. Sonoporation experiments can then be carried out with size- and positioncontrolled bubbles, enabling determination of the optimum conditions for sonoporation by understanding its mechanisms.

\section{MATERIALS AND METHODS}

\section{A. Light Microscope}

Figure 1 shows a block diagram of the observation system. 


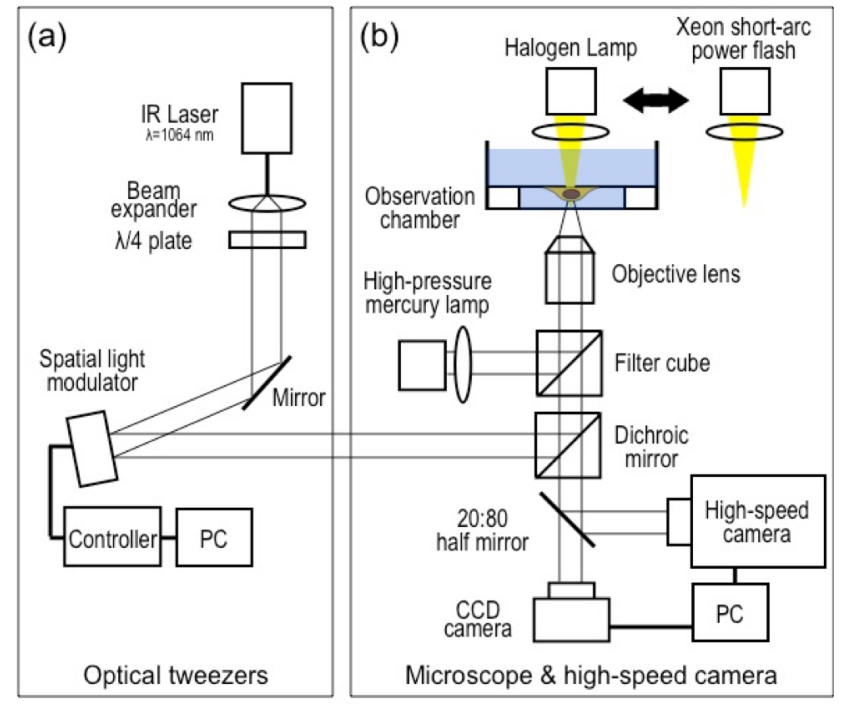

Fig. 1. Block diagram of the observation system. The system consists of (a) optical tweezers and (b) inverted-type fluorescence microscope equipped with a high-speed camera.

The main part of the system is an inverted-type fluorescence microscope. We used two microscopes depending on the purpose of study. One is IX70 (Olympus, Tokyo, Japan), which was used for high-speed observation of bubble dynamics and bubble-cell interaction. It was used for bright field (BF) and fluorescence observation of cell damage. The other is Ti Eclipse (Nikon, Tokyo, Japan), which was used for differential interference contrast (DIC) and fluorescence observation of cell damage. Bubbles and cells were illuminated by a halogen lamp, and a transmission image magnified by an objective lens was captured by a digital CCD (charge coupled device) camera (Orca R2, Hamamatsu Photonics, Shizuoka, Japan). Objective lenses of LUMPlan FI/IR $40 \times$ (N.A. 0.8) and S Plan Fluor ELWD $40 \times$ (N.A. 0.6) were used for IX70 and Ti Eclipse, respectively.

Another light source was used for fluorescence observation. A filter cube with filters and a dichroic mirror was inserted into

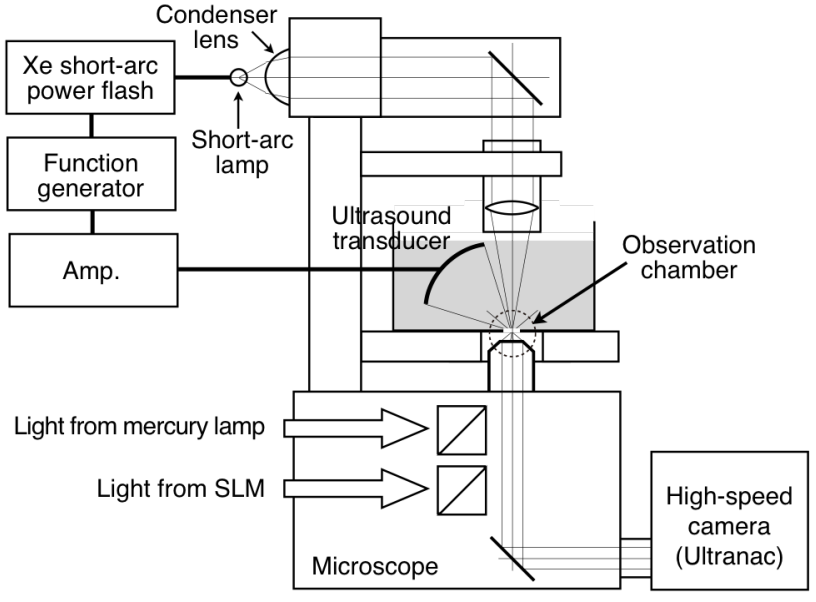

Fig. 2. Schematic view of the observation system configured for high-speed observation. A high-speed camera is attached to a front port of the microscope, and a Xenon short-arc power flash is attached to a lighthouse. An observation chamber is created at the bottom of a water bath placed on the table of the microscope. the optical path to separate excitation light and emission light. The fluorescence dye Propidium iodide (PI) was used to detect cell membrane damage during sonoporation. The dye permeates only through damaged cell membranes and generates $617-\mathrm{nm}$ fluorescence light under excitation of 536-nm light.

\section{B. Observation Chamber}

The observation chamber plays an important role in in situ real-time observation of sonoporation phenomena under the condition of ultrasound exposure. Figure 2 shows the microscope with a water bath placed on its table. The observation chamber was created at the bottom plate of the water bath. As shown in Fig. 3, a hole of about $10 \mathrm{~mm}$ in diameter was drilled through the bottom plate. The bottom side of the hole was covered with a plain coverslip, and the upper side of the hole was covered with a coverslip with a monolayer of cultured cells with cells facing down. The chamber was filled with air-saturated Hanks' balanced salt solution (HBSS) with suspended microbubbles. Bubbles that have risen up to the top of the chamber by buoyancy force can therefore make contact with the cells. For experiments to simulate in vivo sonoporation, stiffness of a culture scaffold can be changed using gels that have stiffness similar to that of biological tissue [20].

Human epithelial prostate adenocarcinoma cells (PC-3 cells) were obtained from American Type Culture Collection. The cells were seeded on a coverslip placed inside a petri dish and incubated at $37^{\circ} \mathrm{C}$ with $5 \% \mathrm{CO}_{2}$ RPMI 1640 medium. Cell samples were used at a condition of 30-50\% confluence.

Albumin-shelled microbubbles with a size distribution of 2 to $7 \mu \mathrm{m}$ in diameter were used to evaluate the dependency of trapping force on bubble size with a wide range of diameters. For obtaining these bubbles, $50 \mathrm{mg}$ of bovine serum albumin and $50 \mathrm{mg}$ of D-glucose were dissolved in $3 \mathrm{~mL}$ of HBSS, and a vial containing $1 \mathrm{~mL}$ of the solution was mechanically agitated for $45 \mathrm{~s}$. Levovist and Bubble liposomes (BL) [21], which were designed for ultrasound contrast agents, have narrower size distributions of $1 \mu \mathrm{m}$ and $3 \mu \mathrm{m}$ in typical diameters, respectively. Levovist was used for observation of cell-bubble interaction using a trappable bubble with a minimum diameter. $\mathrm{BL}$ were used for experiments to investigate the dependence of

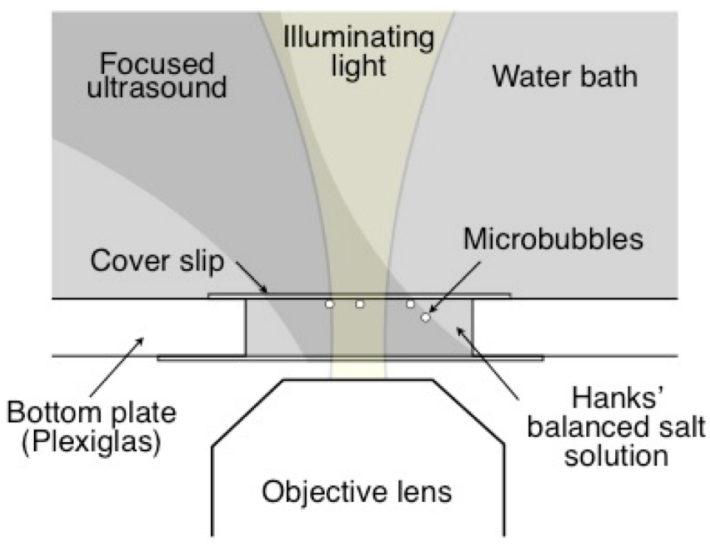

Fig. 3. Observation chamber for in situ microscopic observation of bubble dynamics and bubble-cell interaction. 
the cell ability to repair damage on membrane tension in a typical sonoporation condition in which bubbles of several microns in size were used.

\section{Ultrasound Transducer}

A laboratory-assembled focused transducer of $50 \mathrm{~mm}$ in aperture and $70 \mathrm{~mm}$ in radius that was driven by a three-cycle pulse of $1 \mathrm{MHz}$ in center frequency was used. Two pulses with different pressure amplitudes were used in this study: a pulse with 1.3 and $-0.7 \mathrm{MPa}$ and a pulse with 0.7 and $-0.5 \mathrm{MPa}$ in peak positive and negative pressures, respectively. The pressure waveforms were measured using a membrane hydrophone (MHA500B, NTR Systems, Seattle, WA), which was placed at the focus of the ultrasound transducer in the absence of the observation chamber [8]. An imbalance in peak positive and negative pressure amplitudes indicates the induction of non-linear propagation.

\section{High-speed Camera}

A high-speed camera (Ultranac, Nac Image Technology, Tokyo, Japan) that can operate at a framing rate up to 20 million frames per second (fps) with 24 frames was used in the observation system. The camera uses a computer-controlled image converter tube, which enables independent settings of framing condition such as delay, exposure, and interframe times. Captured images are converted into electron images and deflected by two pairs of orthogonal deflection electrodes so as to make a two-dimensional (2D) matrix of electron image on a phosphor screen. The 2D image matrix is then converted into an optical image matrix and recorded on an instant film.

The camera was attached to a front port of the IX70 microscope, and BF illumination was used for high-speed framing to secure sufficient light exposure. A Xenon short-arc power flash (SA-200S, Nissin Electronic, Tokyo, Japan) was used as an illuminating light source of high-speed imaging. Input electric power of the lamp was 50-100 J per flash. The arc lamp was replaced with a halogen light bulb of a standard illuminating lighthouse. The frame rate was set in the range of 4-16 Mfps for practical observation, and exposure time for one frame was varied in the range of $23-200 \mathrm{~ns}$.

\section{E. Optical Tweezers}

Optical tweezers manipulate a transparent object by reaction

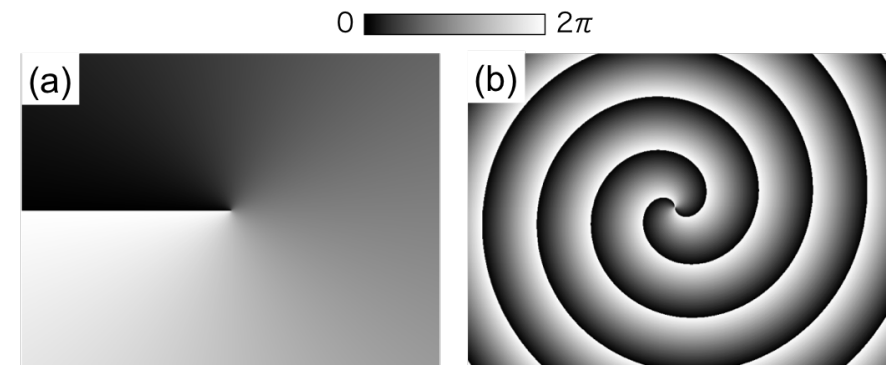

Fig. 4. Holograms used to generate donut light beams for optical tweezing of a microbubble. Holograms for (a) Laguerre-Gaussian (LG) beam ( $l=1)$ and (b) Bessel beam $(l=2)$. force generated by change in momentum of a high-intensity light beam refracted by the object. A microbubble has a refractive index lower than that of surrounding water and receives repulsive force from the beam; therefore, optical tweezers use a donut-shaped beam for bubble trapping [22].

The optical system for the tweezers is also shown in Fig. 1. The tweezers were installed in both of the microscopes. A continuous wave laser of 1,064 $\mathrm{nm}$ in wavelength and $2 \mathrm{~W}$ in maximum optical power was used for a light source. A computer-generated hologram was displayed on the liquid crystal device $(800 \times 600$ pixels $)$ of a reflection-type phase-only spatial light modulator (SLM; X10468, Hamamatsu photonics, Shizuoka, Japan), and a collimated beam of $8 \mathrm{~mm}$ in diameter illuminated the hologram. A phase-modulated light beam was introduced into the optical pathway of the microscope using a dichroic mirror that reflects 1,064-nm light.

Two types of tweezing beams with different beam shapes were used for the experiments. Figure 4a shows hologram for a Laguerre-Gaussian (LG) beam calculated using (1) [23]:

$$
T_{\mathrm{LG}}(r, \theta)=e^{i l \theta}
$$

where $l$ is the topological charge of the phase singularity, $r$ and $\theta$ are the radius and angle measured in the plane transverse to the direction of light propagation, respectively. Figure $4 \mathrm{~b}$ shows hologram for Bessel beam calculated using (2) [24]:

$$
T_{\text {Bessel }}(r, \theta)=e^{i l \theta} e^{-i 2 \pi u x} e^{-i 2 \pi r / r_{0}},
$$

where $u$ is the spatial frequency, $x$ is the distance along the $x$ axis, and $r_{\mathrm{o}}$ is the adjustable constant parameter, respectively. Since the collimated beam is focused by the objective lens with a large numerical aperture, the tweezing beams have a cone shape in $3 \mathrm{D}$ space. The diameter of the donut beam can be adjusted to a bubble size by moving the focus position of the microscope.

Transverse trapping forces of the tweezers were estimated by measuring the maximum moving speeds of trapped bubbles. A bubble moving through water receives two transverse forces in opposite directions: trapping force of the tweezers and viscosity drag force from the surrounding water. Since these two forces become equal at the maximum trapping speed, dragging force $D$ of the optical tweezers can be obtained using (3):

$$
D=6 \pi \mu r_{\mathrm{B}} v,
$$

where $\mu$ is absolute viscosity, $r_{\mathrm{B}}$ is bubble radius, and $v$ is the maximum speed of a dragged bubble.

\section{F. Control of Sonoporation Conditions Using Optical Tweezers}

Sonoporation of biological cells consists of two consecutive processes: membrane rupture and its resealing. Cells have two mechanisms to repair the damage: one is passive resealing of a lipid bilayer and the other is an active resealing called patch 

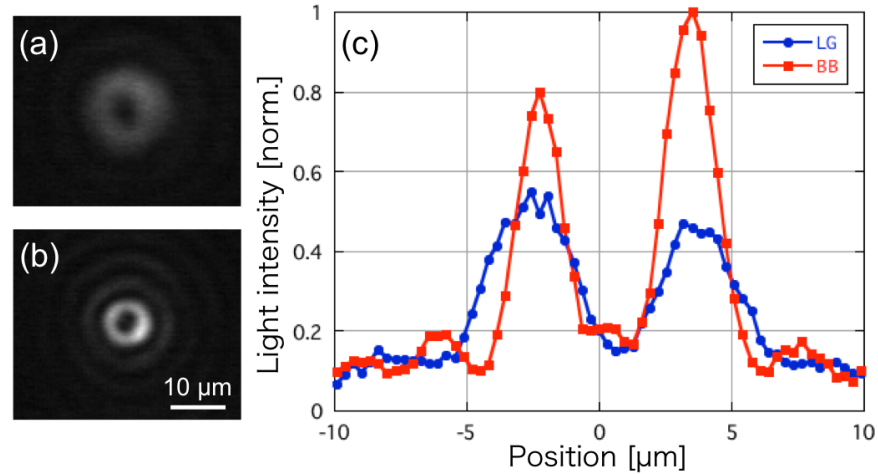

Fig. 5. LG and Bessel beams generated by the optical tweezers. (a) LG beam and (b) Bessel beam. The inner diameters of the beams are adjusted for trapping of the same bubble. (c) Intensity profiles of the beams.

theory [25], [26]. In both resealing mechanisms, cell membrane tension can be an important factor that determines the speed and success rate of membrane resealing.

To confirm the effect of cell membrane tension on resealing, a series of sonoporation experiments was carried out using two types of cells with different shapes [27]: spherical and spindle-shaped cells, which were selected from sparsely cultured monolayer cells. The spherical cells were partially adhered to a coverslip, and the spindle-shaped cells were tightly adhered to a coverslip with extension of filopodia. We hypothesized that the spindle-shaped cells have membrane tension higher than that of the spherical cells. A membrane is lined with the underlying cytoskeleton. When the membrane is disrupted, the cytoskeleton creates membrane tension in the direction to expand the disruption [25]. We considered that cells with spindle shapes create higher tension because the cells have well-developed unidirectional structure of cytoskeleton to keep the stretched cell shapes.

To unify sonoporation conditions at the single cell level, a microbubble of desired size was trapped using the optical tweezers to attach it to intended position of the target cell, and other bubbles around the cell were moved to a distance of about 100-200 $\mu \mathrm{m}$ from the cell so as not to disturb the dynamics of the bubble beside the target cell.

\section{RESULTS}

\section{A. Bubble Trapping}

LG and Bessel beams generated by the optical tweezers installed in the IX70 system are shown in Figs. 5a and 5b, respectively. Figure $5 \mathrm{c}$ shows optical intensity profiles of the
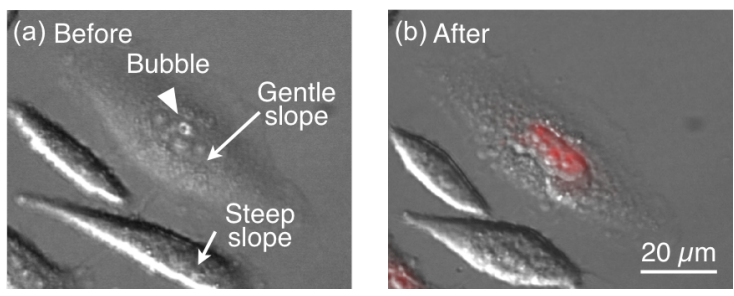

Fig. 7. Position control of a microbubble using the optical tweezers. (a) A 2- $\mu \mathrm{m}$ albumin-shelled bubble that was on the coverslip surface is moved to the cell top (arrowhead) using the Bessel beam. (b) After exposure to one-shot pulsed ultrasound of $0.7 \mathrm{MPa}$ in peak negative pressure. Red color shows propidium iodide (PI) fluorescence.


Fig. 6. Bubble trapping forces of the LG and Bessel beams measured using albumin-shelled microbubbles. The forces linearly increase with increase in bubble diameter.

beams measured at the setting of the same inner diameters of the beams, indicating that the peak intensity of the Bessel beam is 1.8-times higher than that of the LG beam.

The three photos in Fig. 6a shows a bubble, the LG beam, and the bubble trapped by the LG beam from the top, respectively. Figure $6 \mathrm{~b}$ shows the results of transverse trapping force measurements at the optical power of $1 \mathrm{~W}$ of laser output. Albumin-shelled bubbles of 3 to $7 \mu \mathrm{m}$ in diameter with Brownian motion were used for the measurements. The diameter of the trapping beam was adjusted to the size of the trapped bubble. The experimental results showed that the trapping force linearly increases with increase in bubble diameter, and the force of the Bessel beam is 2.4-times higher than that of the LG beam.

Since the donut beam produces force to move bubbles in a transverse direction, a trapped bubble with Brownian motion moves by tracing the surface of an observation sample. Figure 7 shows an albumin-shelled bubble climbing up to the cell top by optical trapping force. A Ti Eclipse microscope was used to observe fluorescence overlaid DIC images. Figure 7a shows the bubble (arrowhead) moved to the cell top, where the bubble
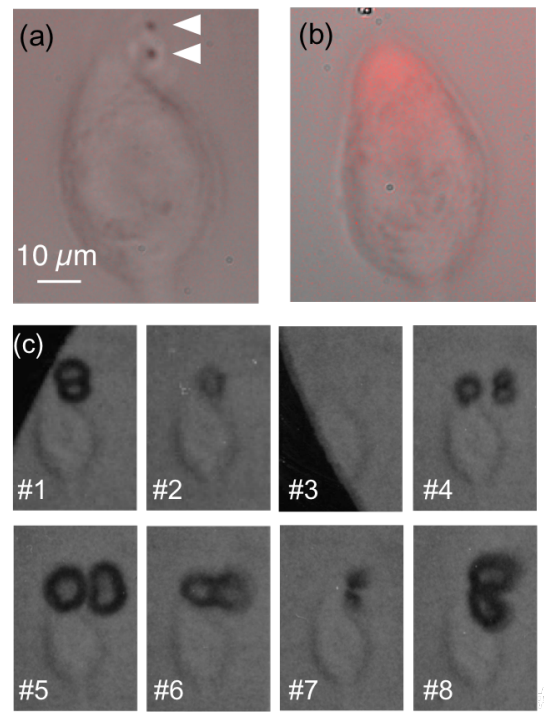

Fig. 8. High-speed and fluorescence observation of bubble dynamics and cell damage. (a) PI fluoresce-overlaid BF image before ultrasound exposure. A cell has two adjacent $1-\mu \mathrm{m}$ bubbles of Levovist. (b) Image after exposure to one-shot pulsed ultrasound of $0.5 \mathrm{MPa}$ in peak negative pressure. (c) High-speed frames taken at the framing rate of 4 million fps. 
cannot keep the position without trapping force. The bubble can reach the cell top when it traces a gentle slope but cannot reach the cell top when it traces a steep slope shown in Fig. 7a. Figure $7 \mathrm{~b}$ shows the cell after exposure to single-shot pulsed ultrasound of $0.7 \mathrm{MPa}$ in peak negative pressure. Appearance of PI fluorescence at the bubble location indicates that sonoporation of a target cell can be achieved by using a sizeand position-controlled bubble.

\section{B. High-speed and Fluorescence Observations}

Figure 8a shows an overlay of BF and florescence images of a cell before ultrasound exposure. Two 1- $\mu \mathrm{m}$ bubbles of Levovist (arrowheads) were trapped by an individual beam generated utilizing a time-division multiplexing technique with two holograms. No fluorescence is observed before ultrasound exposure. Figure 8c shows high-speed frames taken by the IX70 system. Bubble-cell interaction under exposure to single-shot pulsed ultrasound of $0.5 \mathrm{MPa}$ in peak negative pressure was captured at 4 million fps. Frames \#1-\#3 show coalescence of two bubbles in a vertical line followed by bubble contraction. Frames \#4-\#6 show expansion and coalescence of two bubbles in a horizontal line. Frames \#7-\#8 show appearance of two bubbles again in a vertical line, suggesting generation of a vertical liquid flow in Frame \#3 and a horizontal liquid flow in Frame \#7. In Fig. 8b, after ultrasound exposure, PI fluorescence was observed at the location of bubble fragmentation, indicating that the violent volumetric change of the bubbles and/or the resulting liquid flows can cause cell membrane rupture.

\section{Control of Sonoporation Conditions using the Optical Tweezers}

Figure 9 shows images of the sonoporation of cells with spherical and spindle shapes that were taken by using the $\mathrm{Ti}$ Eclipse microscope with the optical tweezers. Figures 9a and $9 \mathrm{~b}$ are DIC images of the cells before sonoporation. BL bubbles were picked up by the optical tweezers and placed beside the
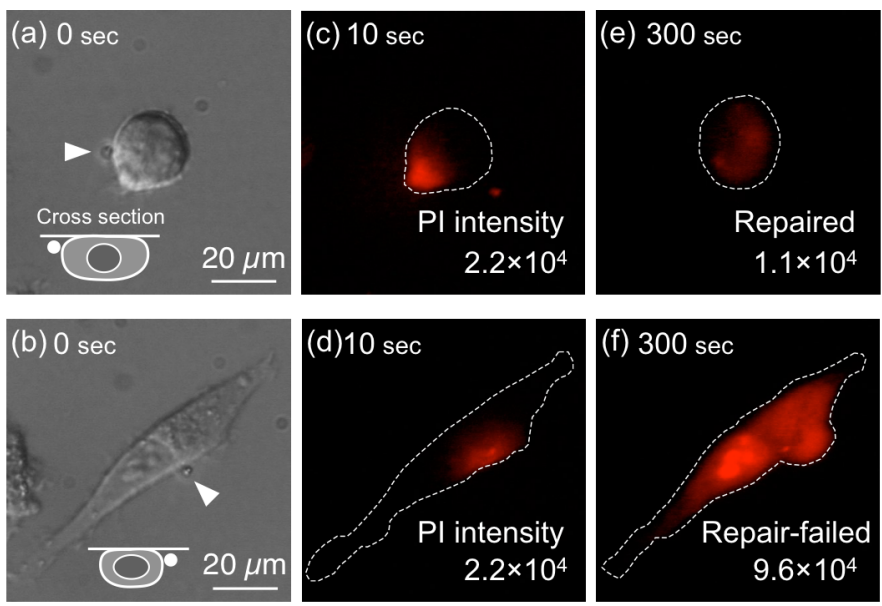

Fig. 9. Sonoporation of cells with spherical and spindle shapes. (a), (b) DIC images before ultrasound exposure to one-shot pulsed ultrasound of $0.5 \mathrm{MPa}$ in peak negative pressure. (c), (d) PI fluorescence images $10 \mathrm{~s}$ after exposure and (e), (f) $300 \mathrm{~s}$ after exposure. White dotted lines in (c)-(f) show shapes of the cells observed by DIC images. nuclei of the cells (arrowheads). The projected areas (Mean \pm S.D.) of spherical and spindle-shaped cells were $385 \pm 84 \mu \mathrm{m}^{2}$ and $673 \pm 184 \mu \mathrm{m}^{2}$, respectively. A significant difference between these values $(p<0.01)$ suggests that the spindle-shaped cells have higher membrane tension than that of spherical cells.

A single-shot ultrasound pulse of $0.5 \mathrm{MPa}$ in peak negative pressure was irradiated to the cells, and temporal change of the cells was studied using time-lapse observation. Figures $9 \mathrm{c}$ and $9 \mathrm{~d}$ show PI fluorescence images $10 \mathrm{~s}$ after ultrasound exposure. Outlines of the cells visualized using DIC images are indicated by dotted lines. In both results, red fluorescence was observed adjacent to the bubble position, indicating membrane damage was generated by direct mechanical activity of the bubble. In these images, integrated fluorescence intensities inside the cells have the same value, $2.2 \times 10^{4}$ (arbitrary units).

Fluorescence images taken $300 \mathrm{~s}$ after sonoporation are shown in Figs. 9e and 9f. In the case of a spherical cell, it was observed that weak fluorescence spread into a cell (Fig. 9e). The integrated fluorescence intensity was decreased to $1.1 \times 10^{4}$, suggesting that resealing of the cell membrane damage was completed immediately after sonoporation. In the case of a spindle-shaped cell, cell swelling was observed beside the initial bubble position (Fig. 9f). Strong fluorescence filled most of the cell, and the integrated fluorescence intensity was increased to $9.6 \times 10^{4}$, indicating failure of membrane resealing.

Changes in PI fluorescence intensity during sonoporation were observed for 27 spherical cells and 30 spindle-shaped cells, and the results are summarized in Figs. 10a and 10b, respectively. In both graphs, the horizontal axis shows bubble size and the vertical axis shows integrated fluorescence intensity $300 \mathrm{~s}$ after sonoporation. In Fig. 10a, closed and open circles show individual repair-failed and repaired cells with a spherical shape. In Fig. 10b, closed and open diamonds show individual repair-failed and repaired cells with a spindle shape.
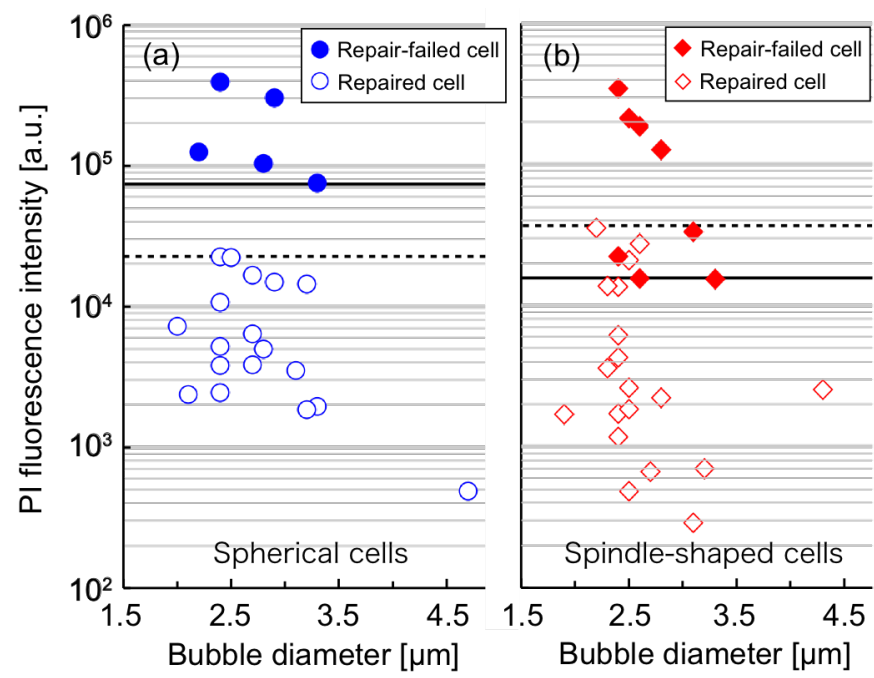

Fig. 10. Sonoporation-induced cell damage was evaluated by PI fluorescence intensity that appeared inside the cells. (a) Spherical cells. (b) Spindle-shaped cells. Solid and dotted lines in each graph represent the minimum intensity level to cause lethal damage and maximum intensity level at which the membrane can be repaired, respectively. 
Repair-failed and repaired cells were categorized by using the results of time-lapse observation of PI fluorescence from $10 \mathrm{~s}$ to $300 \mathrm{~s}$ : repair-failed cells have an integrated fluorescence intensity that continuously increases, while repaired cells have an integrated fluorescence intensity that temporally increases but soon stops.

Rates of cell membrane damage detected by appearance of PI fluorescence were $85 \%(23 / 27)$ in spherical cells and $93 \%$ $(26 / 30)$ in spindle-shaped cells, indicating that the presence of an adjacent bubble at the controlled position greatly increases the rates of cell membrane damage.

Generally, repair-failed cells had higher fluorescence intensities than those of the repaired cells. Bubbles of 1.8-4.3 $\mu \mathrm{m}$ in diameter were used for the experiments; however, no clear correlation was found between fluorescence intensity and bubble size. Rates of repaired cells were $78 \%(18 / 23)$ in spherical cells and 70\% (19/27) in spindle-shaped cells, indicating that sonoporation with bubble control can achieve repair rate higher than that of $\sim 60 \%$ attained in the experiments without bubble control [8].

Solid and dotted horizontal lines in Figs. 10a and 10b show lethally damaged cells with minimum fluorescence intensity (minimum level to cause lethal damage) and repaired cells with maximum fluorescence intensity (maximum level of repairable damage), respectively. As shown in the graphs, the two cell groups have similar maximum levels of repairable damage. However, the minimum level to cause lethal damage in the spherical cell group was 5-times higher than that in the spindle-shaped cell group, indicating that cells with lower membrane tension may have greater ability for repair.

\section{DISCUSSION}

\section{A. Advantage and Limitation of Optical Tweezers}

Microbubbles observed in the sonoporation experiments can be divided into two bubble groups with and without Brownian motion. Bubbles in the former group are floating beneath a coverslip or a cell by forces such as electric repulsion, and they can be moved by the optical tweezers. Bubbles in the latter group directly adhere to the coverslip or cell, and they cannot be moved by the optical tweezers. The ratio between the former and latter bubbles depends on the material of the bubble shell and scaffold surface.

Beam steering and multi-beam operations are possible as shown in Fig. 8a by just substituting a hologram displayed on the SLM. However, trapping forces were measured using a single static beam (Fig. 5a and 5b) because modification of the holograms causes a decrease in trapping force. The trapping force of the single static beam was the same or greater than that generated by a donut beam made by mechanical light beam scan [28], and it was sufficient for transverse movement of bubbles with sizes of several microns that were used for sonoporation experiments.

The buoyancy force of a 3- $\mu \mathrm{m}$ bubble is calculated to be in the order of $0.1 \mathrm{pN}$. Although the transverse trapping force of our system ( $15 \mathrm{pN}$ for the $3-\mu \mathrm{m}$ bubble) is sufficiently larger than the buoyancy force, it was not effective for trapping in the vertical direction. Three-dimensional (3D) trapping of a microbubbles has been achieved in several studies. An upright microscope was typically used in those studies [28], [29], and position control in a vertical direction was realized by balancing two forces that work in opposite directions: optical radiation force and bubble buoyancy force. The first sonoporation experiment using an optically trapped bubble [16] also used an upright microscope, and the bubble was trapped to float in the water at a distance from a cell cultured on a coverslip. Our observation system uses an inverted-type microscope, and optical and buoyancy forces work in the same direction. Hermerschmidt proposed a method for the 3D trapping [30], which is our forthcoming task.

Sonoporation of Xenopus oocytes with 3D trapping bubbles generated by laser induced optical break down was also studied [31]. A high-speed camera was used to visualize bubble dynamics beside the cells, and resulting membrane damage was evaluated by measurements of transmembrane current.

\section{B. High-speed observation}

The complexity of microbubble dynamics under the condition of ultrasound exposure drastically increases in the presence of neighboring bubbles and cells. The reason is that interactions between the bubbles and cells cause anisotropic oscillation and translational movement of bubbles that lead to bubble fragmentation and cell deformation. Not only a higher framing rate but also a larger frame number is needed to understand the complex bubble dynamics. Brandaris-128 [32], which can take 128 frames at 25 million fps, has been used for a wide range of studies on medical application of microbubbles. The maximum number of frames of our camera is limited to 24 frames; however, the ability for independent setting of exposure time and framing interval allows study on the effect of a shell material on bubble dynamics, with observation of bubble dynamics under five sequential pulses with increasing pressure amplitudes [33]. High-contrast observation of a biological cell is also important for high-speed studies on bubble-cell interaction. High-image quality is one of the advantages of a high-speed camera that uses a sensitive CMOS image sensor with 128 on-chip memories [34].

\section{BF, DIC and fluorescence observations}

$\mathrm{BF}$ and DIC observations before sonoporation were important to select bubbles and cells for controlling sonoporation conditions at the single cell level, and observation of PI fluorescence after sonoporation was important as a sensitive indicator for evaluating the degree of membrane damage. Furthermore, resealing of the damaged membrane was determined by evaluation of temporal change in PI fluorescence, indicating usefulness of in situ time-lapse observation in sonoporation studies using size- and position-controlled bubbles.

The BF images visualized changes in shape and contrast of cells after sonoporation (Fig. 8). The DIC images showed that 
local membrane damage initiates change in the texture of the entire cell (Fig. 9). However, it is still difficult to visualize 3D changes in a cell, and therefore, confocal microscopy is important for detecting changes in the membrane and organelles [35]-[37].

\section{Dependence of Membrane Resealing on Membrane Tension}

The cell membrane has an inherent ability to reseal a small pore by fusion of the disrupted edge of the pore. In the case of a large liposome, the resealing process of pores up to $1 \mu \mathrm{m}$ in diameter is finished in a time scale of milliseconds to seconds, and lower membrane tension causes more rapid resealing [38]. Feril et al. [39] reported that ultrasonically induced membrane damage of cells suspended in a hypotonic medium is greater than that of cells suspended in a normal medium. The results suggest that cell swelling and the resulting increase in membrane tension prevented the cells from resealing.

These reports support our experimental results that cells with lower membrane tension have higher ability to repair sonoporation damage. This finding is important for studies toward realization of in vivo sonoporation because the difference in cell morphology and tissue stiffness in in vitro and in vivo situations causes a significant difference in the persistence of cells against ultrasound exposure [20].

\section{CONCLUSION}

At the microscopic level, damage and repair of the cell membrane during sonoporation have large variance at the individual cell level because the cell shape, bubble size, and their positional relationship have large variance. Our approach by which single cell sonoporation is observed therefore has a limitation, and a statistical approach by which a large number of cells is observed is important to find a general tendency of sonoporation phenomena. On the other hand, averaging in a statistical approach would sometimes mask an important nature of sonoporation. The system used in this study was designed to combine a light microscope with optical tweezes. This combination enables control of sonoporation condition at single cell level and close observation of bubble and cell dynamics induced by sonoporation. We believe that the system will accelerate studies on sonoporation mechanisms to realize in vivo sonoporation.

\section{REFERENCES}

[1] H. J. Kim, J. F. Greenleaf, R. R. Kinnick, J. T. Bronk, and M. E. Boander, "Ultrasound-mediated transfection of mammalian cells," Hum. Gene Ther., vol. 7, pp. 1339-1346, 1996.

[2] C. Newman, A. Lawrie, A. Brisken, and D. Cumberland, "Ultrasound gene therapy: on the road from concept to reality," Echocardiography, vol. 18, no. 4, pp. 339-47, May 2001.

[3] D. L. Miller, S. V Pislaru, and J. E. Greenleaf, "Sonoporation: mechanical DNA delivery by ultrasonic cavitation," Somat. Cell Mol. Genet., vol. 27, no. 1-6, pp. 115-34, Nov. 2002.

[4] A. Lawrie, A. F. Brisken, S. E. Francis, D. C. Cumberland, D. C. Crossman, and C. M. Newman, "Microbubble-enhanced ultrasound for vascular gene delivery," Gene Ther., vol. 7, no. 23, pp. 2023-7, Dec. 2000.

[5] W. J. Greenleaf, M. E. Bolander, G. Sarkar, M. B. Goldring, and J. F. Greenleaf, "Artificial cavitation nuclei significantly enhance acoustically induced cell transfection," Ultrasound Med. Biol., vol. 24, no. 4, pp. 587-595, 1998.

[6] K. Okada, N. Kudo, K. Niwa, and K. Yamamoto, "A basic study on sonoporation with microbubbles exposed to pulsed ultrasound," $J$. Med. Ultrason., vol. 32, no. 1, pp. 3-11, 2005.

[7] K. Okada, N. Kudo, T. Kondo, and K. Yamamoto, "Contributions of mechanical and sonochemical effects to cell membrane damage induced by single-shot pulsed ultrasound with adjacent microbubbles," J. Med. Ultrason., vol. 35, no. 4, pp. 169-176, 2008.

[8] N. Kudo, K. Okada, and K. Yamamoto, "Sonoporation by single-shot pulsed ultrasound with microbubbles adjacent to cells," Biophys. J., vol. 96, no. 12, pp. 4866-4876, 2009.

[9] M. Miller, D. Miller, and A. Brayman, "A review of in vitro bioeffects of inertial ultrasonic cavitation from a mechanistic perspective," Ultrasound Med. Biol., vol. 22, no. 9, pp. 1131-1154, 1996.

[10] M. Wan and Y. F. Gail, Cavitation in Biomedicine. Springer Netherlands. 2015.

[11] N. De Jong, P. J. A. Frinking, A. Bouakaz, M. Goorden, T. Schourmans, X. Jingping, and F. Mastik, "Optical imaging of contrast agent microbubbles in an ultrasound field with a $100-\mathrm{MHz}$ camera," Ultrasound Med. Biol., vol. 26, no. 3, pp. 487-492, 2000.

[12] M. Postema, A. Van Wamel, C. T. Lancée, and N. De Jong, "Ultrasound-induced encapsulated microbubble phenomena," Ultrasound Med. Biol., vol. 30, no. 6, pp. 827-840, 2004.

[13] H. J. Vos, B. Dollet, M. Versluis, and N. De Jong, "Nonspherical Shape Oscillations of Coated Microbubbles in Contact With a Wall," Ultrasound Med. Biol., vol. 37, no. 6, pp. 935-948, 2011.

[14] A. van Wamel, K. Kooiman, M. Harteveld, M. Emmer, F. J. ten Cate, M. Versluis, and N. de Jong, "Vibrating microbubbles poking individual cells: drug transfer into cells via sonoporation," J. Control. Release, vol. 112, no. 2, pp. 149-55, May 2006.

[15] C.-D. Ohl, M. Arora, R. Ikink, N. de Jong, M. Versluis, M. Delius, and D. Lohse, "Sonoporation from jetting cavitation bubbles," Biophys. J., vol. 91, no. 11, pp. 4285-95, Dec. 2006.

[16] P. Prentice, A. Cuschieri, K. Dholakia, M. Prausnitz, and P. Campbell, "Membrane disruption by optically controlled microbubble cavitation," Nat. Phys., vol. 1, no. 2, pp. 107-110, Oct. 2005

[17] K. Kooiman, M. Foppen-Harteveld, A. F. W. Van Der Steen, and N. De Jong, "Sonoporation of endothelial cells by vibrating targeted microbubbles," J. Control. Release, vol. 154, no. 1, pp. 35-41, 2011.

[18] G. Lajoinie, I. De Cock, C. C. Coussios, I. Lentacker, S. Le Gac, E. Stride, and M. Versluis, "In vitro methods to study bubble-cell interactions: Fundamentals and therapeutic applications," Biomicrofluidics, vol. 10, no. 1, p. 011501, Jan. 2016.

[19] N. Kudo, Y. Tanaka, and K. Uchida, "Basic studies on sonoporation with size- and position-controlled microbubbles adjacent to cells," IEEE Int. Ultrason. Symp. IUS, no. c, pp. 53-56, 2013.

[20] N. Kudo and Y. Kinoshita, "Effects of cell culture scaffold stiffness on cell membrane damage induced by sonoporation," J. Med. Ultrason., vol. 41, pp. 1-10, 2014.

[21] R. Suzuki, T. Takizawa, Y. Negishi, N. Utoguchi, and K. Maruyama, "Effective gene delivery with novel liposomal bubbles and ultrasonic destruction technology," Int. J. Pharm., vol. 354, no. 1-2, pp. 49-55, 2008

[22] V. Garbin, "Optical tweezers for the study of microbubble dynamics in ultrasound," Universit'a degli Studi di Trieste, Trieste, Italy, 2006. H. He, N. R. Heckenberg, and H. Rubinsztein-Dunlop, "Optical Particle Trapping with Higher-order Doughnut Beams Produced Using High Efficiency Computer Generated Holograms," J. Mod. Opt., vol. 42, no. 1, pp. 217-223, Jan. 1995.

[24] J. Arlt and K. Dholakia, "Generation of high-order Bessel beams by use of an axicon," Opt. Commun., vol. 177, no. 1, pp. 297-301, 2000.

[25] P. L. Mcneil and T. Kirchhausen, "An emergency response team for membrane repair," Nat. Rev. | Mol. CELL Biol., vol. 6, no. June, pp. 499-505, 2005.

[26] I. Lentacker, I. De Cock, R. Deckers, S. C. De Smedt, and C. T. W. Moonen, "Understanding ultrasound induced sonoporation: Definitions and underlying mechanisms," Adv. Drug Deliv. Rev., vol. 72, pp. 49-64, 2014. 
Y. Tanaka and N. Kudo, "Relation between cell membrane tension and repair of membrane damaged during sonoporation," IEEE Int. Ultrason. Symp. IUS, pp. 1777-1780, 2013.

[28] P. H. Jones, E. Stride, and N. Saffari, "Trapping and manipulation of microscopic bubbles with a scanning optical tweezer," Appl. Phys. Lett., vol. 89, no. 8, pp. 87-90, 2006.

[29] V. Garbin, D. Cojoc, E. Ferrari, E. Di Fabrizio, M. L. J. Overvelde, S. M. van der Meer, N. de Jong, D. Lohse, and M. Versluis, "Changes in microbubble dynamics near a boundary revealed by combined optical micromanipulation and high-speed imaging," Appl. Phys. Lett., vol. 90, no. 11, p. 114103, Mar. 2007.

[30] A. Hermerschmidt, S. Krüger, T. Haist, S. Zwick, M. Warber, and W. Osten, "Holographic optical tweezers with real-time hologram calculation using a phase-only modulating LCOS-based SLM at 1064 nm," Complex Light Opt. Forces II, vol. 6905, pp. 690508-10, Jan. 2008.

[31] Y. Zhou, K. Yang, J. Cui, J. Y. Ye, and C. X. Deng, "Controlled permeation of cell membrane by single bubble acoustic cavitation," $J$ Control. Release, vol. 157, no. 1, pp. 103-111, 2012.

[32] C. T. Chin, C. Lancée, J. Borsboom, F. Mastik, M. E. Frijlink, N. De Jong, M. Versluis, and D. Lohse, "Brandaris 128: A digital 25 million frames per second camera with 128 highly sensitive frames," Rev. Sci. Instrum., vol. 74, no. 12, pp. 5026-5034, 2003.

[33] N. Kudo, K. Okada, and K. Yamamoto, "Study on an evaluation method of shell characteristics of ultrasound contrast agent using a high-speed camera," in IEEE International Ultrasonics Symposium, IUS, 2003, pp. 1507-1510.

[34] R. Kuroda, Y. Tochigi, K. Miyauchi, T. Takeda, H. Sugo, F. Shao, and S. Sugawa, "A 20Mfps global shutter CMOS image sensor with improved light sensitivity and power consumption performances," ITE Trans. Media Technol. Appl., vol. 4, no. 2, pp. 149-154, 2015.

[35] W. Zhong, W. H. Sit, J. M. F. Wan, and A. C. H. Yu, "Sonoporation induces apoptosis and cell cycle arrest in human promyelocytic leukemia cells," Ultrasound Med. Biol., vol. 37, no. 12, pp. 2149-59, Dec. 2011

[36] R. S. Leow, J. M. F. Wan, and A. C. H. Yu, "Membrane blebbing as a recovery manoeuvre in site-specific sonoporation mediated by targeted microbubbles.," J. R. Soc. Interface, vol. 12, no. 105, p. 20150029-, 2015.

[37] I. De Cock, E. Zagato, K. Braeckmans, Y. Luan, N. de Jong, S. C. De Smedt, and I. Lentacker, "Ultrasound and microbubble mediated drug delivery: Acoustic pressure as determinant for uptake via membrane pores or endocytosis," J. Control. Release, vol. 197, pp. 20-28, 2015

[38] D. V. Zhelev and D. Needham, "Tension-stabilized pores in giant vesicles: determination of pore size and pore line tension," $B B A$ Biomembr., vol. 1147, no. 1, pp. 89-104, 1993.

[39] L. B. Feril, K. Tachibana, T. Kondo, R. Ogawa, Q.-L. Zhao, K. Yamaguchi, K. Ogawa, H. Endo, Y. Irie, and Y. Harada, "Hypotonia-induced cell swelling enhances ultrasound-induced mechanical damage to cancer cells," J. Med. Ultrason., vol. 37, no. 1, pp. 3-8, Oct. 2009.

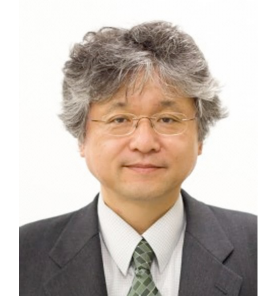

Nobuki Kudo (M'82) was born in Hokkaido, Japan, in 1957. He received a B.S. degree in electronics engineering from Hokkaido University, Sapporo, Japan in 1982 and M.S. and Ph.D. degrees in bioengineering from Hokkaido University in 1984 and 1987, respectively.

From 1987 to 1995, he belonged to Toshiba Cooperation and was engaged in the development of an extra-corporeal shockwave lithotripter. In 1995, he acquired the position of Research Associate in Faculty of Engineering, Hokkaido University and became Associate Professor of the Graduate School of Information Science and Technology, Hokkaido University in 2009. His research interest is application of ultrasound and optical techniques for medical engineering. He is a board member of the Japan Society on Ultrasound in Medicine. He has roles as associate editor of IEEE trans. on UFFC and area editor of Journal of Medical Ultrasonics. 